\title{
ROHON-BEARD NEURONS ARISE FROM A SUBSTITUTE ANCESTRAL CELL AFTER REMOVAL OF THE CELL FROM WHICH THEY NORMALLY ARISE IN THE 16-CELL FROG EMBRYO ${ }^{1}$
}

\author{
MARCUS JACOBSON
}

Department of Anatomy, University of Utah College of Medicine, Salt Lake City, Utah 84132

\begin{abstract}
In 16-cell Xenopus embryos, horseradish peroxidase (HRP) was injected into blastomere D1.2 on one side. No Rohon-Beard neurons originated from D1.2 in either of the two patterns of cleavage that were studied (Jacobson, M. (1981) J. Neurosci. 1: 918-922). In other embryos, after injection of HRP into D1.2, the neighboring ventral blastomere V1.2, from which 68 to $90 \%$ of Rohon-Beard neurons normally originate, was removed. In the cases that developed normally to larval stages 32 to 34 , the number and sizes of Rohon-Beard neurons were normal, but 14.9 to $73.9 \%$ of Rohon-Beard neurons were labeled, showing that they originated from the injected blastomere D1.2. Labeling also occurred in cells of the spinal dorsal root ganglia that normally descend from V1.2 but not from D1.2. This proves that individual blastomeres at the 16-cell stage are not committed to form specific types of neurons or restricted parts of the central nervous system.
\end{abstract}

In the foregoing paper (Jacobson, 1981), it was shown that the majority of Rohon-Beard neurons, as well as other kinds of nerve cells in the dorsal spinal cord and dorsal spinal ganglia, arise from blastomere V1.2 on each side of the 16-cell embryo of Xenopus. These experiments were designed to investigate the effects of removal of blastomere V1.2 and to determine whether partial or complete restoration of the number of Rohon-Beard neurons occurs and if so, to trace the origins of those Rohon-Beard neurons.

Three types of experiments were done. First, the effects of removal of blastomere V1.2 were investigated (experiment 1). When it was found that Rohon-Beard neurons as well as all other parts of the central nervous system developed normally after removal of V1.2, experiments were done to ensure that removal of blastomere V1.2 was complete (experiment 2). In experiment 3 , horseradish peroxidase (HRP) was injected into one of the blastomeres neighboring V1.2 (namely D1.2, V1.1, or V2.2) followed by removal of V1.2 to determine which of these was substituting for V1.2 as the principal ancestral cell giving rise to Rohon-Beard neurons. It was found that any one of those cells could partially substitute for V1.2 as a progenitor of the majority of Rohon-Beard neurons. Substitution of V1.1 or V2.2 for V1.2 is of little interest because those blastomeres normally give rise to RohonBeard neurons and those results will not be discussed further. This paper reports a result of greater interest, namely the production of up to $73.9 \%$ of Rohon-Beard

\footnotetext{
' This work was supported by Grant BNS 7913971 from the National Science Foundation.
}

neurons by blastomere D1.2, which normally does not give rise to any Rohon-Beard cells, after removal of blastomere V1.2, which normally gives rise to the majority of Rohon-Beard cells.

\section{Materials and Methods}

The methods of obtaining embryos and of injecting individual blastomeres were the same as those described in the previous paper. The same two types of cleavage pattern, termed $\mathrm{X}$ and $\mathrm{Y}$, were used (Fig. 1 of Jacobson, 1981).

Experiment 1: Removal of blastomere V1.2. At the 16cell stage, a micropipette (tip diameter, $\sim 20 \mu \mathrm{m}$ ) attached to a micrometer syringe was inserted into blastomere $\mathrm{LV}$ 1.2 or RV 1.2 and the blastomere was sucked out slowly over a period of about $30 \mathrm{sec}$, removing the entire cell or leaving a small remnant in some cases. Blockage of the pipette occurred in most cases and it was necessary to withdraw it and repeat the aspiration. This operation was performed on a total of 10 embryos (5 type $Y$ and 5 type X).

Experiment 2: Determination of the completeness of blastomere removal. Horseradish peroxidase (Sigma Type IX; 20\% in distilled water or Steinberg solution, 1 to $2 \mathrm{nl}$ ) was injected by means of a micropipette (tip diameter, $\sim 5 \mu \mathrm{m}$ ) into blastomere LV 1.2 or RV 1.2. After withdrawal of the injection micropipette, 1 to $5 \mathrm{~min}$ was allowed to elapse before the suction micropipette was inserted into the previously injected blastomere and that blastomere was removed as described above. Eight embryos ( 4 type $\mathrm{X}$ and 4 type $\mathrm{Y}$ ) were treated in this way. 
Experiment 3: Determinalion of the ability of blastomere D1.2 to substitute for blastomere V1.2. Intracellular injection of HRP into blastomere D1.2 was done on one side as described above. One to 5 min later, blastomere V1.2 on the same side was removed by suction as described above. These operations were performed on 30 embryos (15 type $\mathrm{X}$ and 15 type $\mathrm{Y}$ ).

In all experiments, the operated embryos were placed in separate Petri dishes in $100 \%$ Steinberg solution plus gentamicin $(10 \mathrm{mg} / \mathrm{liter})$ for 3 to $6 \mathrm{hr}$ and then were transferred to $50 \%$ Steinberg solution and kept at $20^{\circ} \mathrm{C}$ until they developed to larval stages 32 to 34 (Nieuwkoop and Faber, 1967), 40 to $45 \mathrm{hr}$ after the operation.

Animals were fixed and prepared histochemically to show the presence of peroxidase as described previously (Jacobson and Hirose, 1981; Jacobson, 1981). The intracellular brown reaction product, indicating the presence of HRP, will be referred to as "label." The maximum diameter of the cell body of every Rohon-Beard neuron in 1 specimen of experiment 1 and 1 specimen of experiment 3 was measured using a microscope with an ocular fitted with a filar micrometer. Those measurements were used to correct for split cell counting errors (Abercrombie, 1946). All Rohon-Beard neurons were counted on both sides of the spinal cord in 11 specimens (Table II).

\section{Results}

Of the 48 embryos on which I operated, 24 died, 6 developed abnormally and are not considered further, and 18 developed normally to stages 32 to $34 ; 14$ of those 18 were processed well enough to enable measurement and counts of Rohon-Beard neurons to be made (Tables I and II).
Experiment 1. After removal of blastomere V1.2 on one side in 10 embryos, 5 survived normally to stages 32 to 34 and 3 of those were processed well enough to permit counts and measurements of Rohon-Beard neurons to be made. No histological abnormalities were seen in the central nervous system or elsewhere in those specimens and Rohon-Beard neurons were in their normal positions. The mean diameter of Rohon-Beard neurons was 22.1 $\mu \mathrm{m}$ on the operated side and $23.7 \mu \mathrm{m}$ on the unoperated side of 1 embryo. In 3 specimens, the number of RohonBeard cell bodies was as shown in Table II with a mean of 67 on the unoperated side and 66 on the operated side. The diameters of Rohon-Beard cell bodies and the total numbers of Rohon-Beard cells were not significantly different on the two sides of the operated animals or different from the values in intact (injected but not aspirated) animals of the same stages (Jacobson, 1981).

Experiment 2. After injecting HRP into blastomere V1.2 on one side followed by removal of the labeled blastomere in 8 embryos, 4 died and 4 developed normally to stages 32 to 34 . Three of those were processed satis-

TABLE I

Numbers of embryos used in experiments $\mathrm{X}$ and $\mathrm{Y}$ are different cleavage patterns.

\begin{tabular}{|c|c|c|c|c|c|c|}
\hline \multirow{2}{*}{ Experiment } & \multicolumn{2}{|c|}{ Operated } & \multicolumn{2}{|c|}{ Survived } & \multicolumn{2}{|c|}{ Processed } \\
\hline & $\mathrm{x}$ & $Y$ & $x$ & $\mathrm{Y}$ & $\mathrm{X}$ & $\mathrm{Y}$ \\
\hline 1 & 5 & 5 & 3 & 2 & 2 & 1 \\
\hline 2 & 4 & 4 & 2 & 2 & 2 & 1 \\
\hline 3 & 15 & 15 & 6 & 3 & 5 & 3 \\
\hline Total & 24 & 24 & 11 & 7 & 9 & 5 \\
\hline
\end{tabular}

TABLE II

Rohon-Beard neurons at larval stages 32 to 34 in three types of experiments

The three experiments (E1, E2, and E3) are described in the text. $\mathrm{X}$ and $\mathrm{Y}$ are different cleavage patterns. LD, LV, RD, and RV refer to left dorsal, left ventral, right dorsal, and right ventral cells, respectively.

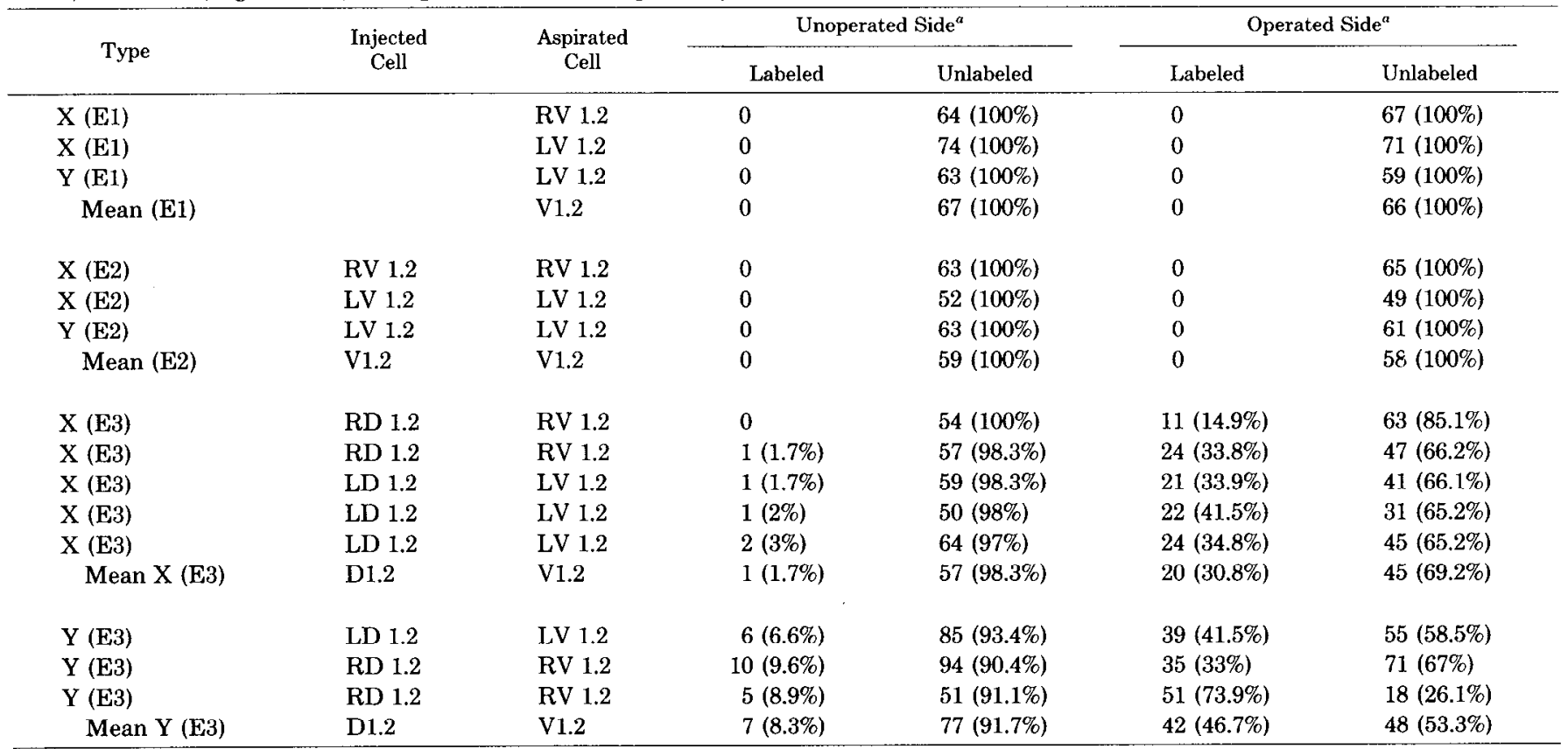

${ }^{a}$ Corrected for split cell counting errors. 
factorily and were normal on histological examination. No labeled cells were seen in those embryos, showing that the initially labeled blastomere had been completely removed. Counts of Rohon-Beard cell bodies (Table II) gave a mean of 59 on the unoperated side and 58 on the operated side. Those numbers are not significantly different from one another or from the numbers of RohonBeard cell bodies in unoperated normal embryos. This shows that normal numbers of Rohon-Beard neurons developed after removal of blastomere V1.2 from which the majority of these cells normally originate.

Experiment 3. We have shown that Rohon-Beard neurons are not labeled after HRP injection of D1.2 (Hirose and Jacobson, 1979) or of its descendants at 32- and 64cell stages (Jacobson and Hirose, 1981). This experiment was designed to show whether Rohon-Beard neurons could descend from blastomere D1.2 in the absence of V1.2. Of 30 embryos in which HRP was injected into blastomere D1.2 followed by aspiration of V1.2 on the same side, 9 survived to stages 32 to 34 (Table I). Of these, 8 were processed successfully and all appeared normal on histological examination. A considerable number ( 14.9 to $73.9 \%$ ) of the labeled Rohon-Beard neurons were present un the operated side in all cases and a small number ( 1.7 to $9.6 \%$ ) of the labeled Rohon-Beard neurons were found on the unoperated side. The mean diameter of the labeled Rohon-Beard cell bodies on the operated side in one specimen was $22.5 \mu \mathrm{m}$ and the mean diameter of the unlabeled Rohon-Beard cell bodies on the operated side in the same specimen was $22.8 \mu \mathrm{m}$. These values are not significantly different from the diameters of RohonBeard cell bodies that descended normally from blastomere V1.2 (Jacobson, 1981). The total numbers of RohonBeard neurons in the operated embryos were within the normal range (Jacobson, 1981; Table I). However, the mean percentages of labeled Rohon-Beard neurons that originated from blastomere D1.2 on the operated side (34.8\% type X; 46.7\% type Y) were about half of the mean percentages of Rohon-Beard neurons (70\% type X; $81 \%$ type Y) that originated from blastomere V1.2 in normal embryos (Jacobson, 1981; Table I). The unlabeled Rohon-Beard neurons on the injected side in these experimental animals probably originated from other blastomeres, such as V1.1 and V2.2, which normally give rise to about $27 \%$ of the Rohon-Beard neurons on the same side.

A small increase was observed in the percentage of labeled Rohon-Beard neurons on the contralateral side in this experiment (1.7\% type $\mathrm{X} ; 8.3 \%$ type $\mathrm{Y}$ ) when compared with the percentages arising from V1.2 contralaterally in normal embryos $(0 \%$ type $\mathrm{X} ; 4 \%$ type $\mathrm{Y}$; Jacobson, 1981; Table I). The most likely explanation of this effect is that aspiration of blastomere $\mathrm{V} 1.2$ resulted in movement of part of the injected blastomere D1.2 across the presumptive plane of bilateral symmetry of the embryo. Geometrical distortions of the embryo always occurred after sucking out blastomere V1.2 so that part of D1.2, normally entirely on one side, could cross the presumptive midline and give rise to Rohon-Beard neurons on both sides of the central nervous system.

In addition to labeled Rohon-Beard neurons, other types of cells that were labeled in all specimens included extramedullary neurons and cells in the dorsal spinal ganglia. 'These cell types normally are labeled after injection of V1.2 but not after injection of D1.2 (Hirose and Jacobson, 1979). The labeled Rohon-Beard neurons were scattered along the length of the spinal cord, interspersed with unlabeled Rohon-Beard neurons.

\section{Discussion}

These experiments provide a new example of regulation in the amphibian embryo, and by focusing on the regulation of Rohon-Beard cells which are a unique neuronal type localized in the dorsal spinal cord, the experiments raise questions about the developmental information that may be required for differentiation of this and other specific types of nerve cells. By showing that blastomere $\mathrm{D} 1.2$, which normally does not give rise to Rohon-Beard neurons, can replace V1.2 as the principal progenitor of Rohon-Beard neurons, these experiments show that there is no developmental information necessary for the differentiation of Rohon-Beard neurons that is uniquely localized in the blastomere which normally gives rise to most of those neurons. As shown in Figure 1, after labeling D1.2 and removing V1.2, the labeled descendants of D1.2 populated the domain normally occupied by descendants of V1.2 in the central nervous system. The Rohon-Beard neurons that descended from D1.2 differentiated in ways appropriate to their positions in the domain of V1.2, not in accordance with the presumptive fate of D1.2 in the normal embryo. Complete regulation of Rohon-Beard neurons, defined as the restoration of normal numbers of normal size Rohon-Beard neurons from alternative progenitors after removal of their principal normal progenitor, occurred.

These results are an advance on previous work on regulation in the amphibian embryo in three main respects. Firstly, the prospective fate of the removed cells was accurately known in terms of a specific type of descendant cell, the Rohon-Beard neuron. Secondly, the cells that replaced the Rohon-Beard neurons could be identified, and thirdly, counts of the number of RohonBeard neurons that were replaced provided a quantitative assay of the amount of regulation.

Although there have been many reports showing the considerable regulative ability of the amphibian blastula (Spemann, 1938; Holtfreter and Hamburger, 1955), they all have been deficient in one or more of the criteria listed above for assaying regulation. A detailed fate map of part of the presumptive nervous system in the amphibian blastula was not available until recently (Jacobson and Hirose, 1981) and the earlier vital staining techniques failed to identify the progenitors of any specific type of neuron, including such easily identifiable neurons as the Rohon-Beard cells (Vogt, 1929; Nakamura and Kishiyama, 1971; Nakamura et al., 1978). In previous studies, the criteria used for assaying the completeness of regulation were merely qualitative and the ancestry of the replaced cells was not known.

The only other study of the effects of removal of identified blastomeres in Xenopus (Nakamura and Takasaki, 1971) found complete absence of the regulation of eyes and ears after removal of the eight blastomeres at the animal pole at the 32 -cell stage. Although the report provided some evidence that the residual blastomeres 
developed autonomously according to their original prospective fates in the intact embryo, insufficient histolog. ical evidence was given and no experiments were reported in which fewer than eight blastomeres were removed. Nevertheless, those results suggest that the eight animal pole blastomeres at the 32-cell stage are uniquely able to give rise to eyes and ears. Other interpretations of those observations may be advanced, yet they raise the general question of the spatial and temporal limitations on replacement of one blastomere by another during regulation. One way of posing the question of replacement of one progenitor by another during regulation is to ask whether boundaries arise between groups of cells during development so that cells on the same side are interconvertible, whereas cells on opposite sides of the boundary cannot replace one another. The present observations, as well as some others showing that V1.2 can partly replace D1.2, show that there is no such boundary between blastomeres D1.2 and V1.2 at the 16-cell stage. We may determine then whether the lineages of D1.2 and of V1.2 remain interconvertible at later stages. This is being done by labeling D1.2 or V1.2 but delaying the removal of some or all of the descendants until later stages.

Is D1.2 able to replace V1.2 because of their similarity in the sense that they are both progenitors of cells in the dorsal spinal cord, many of which originate at about the same time as Rohon-Beard neurons? This was suggested to me by the report by Sulston and White (1980) that, in the embryo of the nematode Caenorhabditis elegans, one cell can replace another during regulation when the cells resemble one another, but cells that are unique are not replaceable. In the amphibians, the only unique presumptive neural cell is D2.2 at the 16-cell stage and its descendants D2.2.2 and D2.2.2.1 at the 32- and 64-cell stages. That lineage gives rise to the Mauthner neuron (Jacobson and Hirose, 1981) and I am doing experiments to determine whether regulation of the Mauthner neuron can occur after removal of D2.2 or its descendants at 32 and 64-cell stages.

Another sort of singularity is seen in the set of ancestral cells that gives rise to the entire population of a specific type of differentiated cell. For example, blastomeres V1.2 and V2.2 in type $\mathrm{Y}$ embryos and V1.1, V1.2, and V2.2 in type $\mathrm{X}$ embryos give rise to all of the Rohon-Beard neurons (Jacobson, 1981) and may be called the set of progenitors of that type of neuron. We do not know yet the results of removal of the entire set of progenitors of Rohon-Beard neurons. These experiments show that

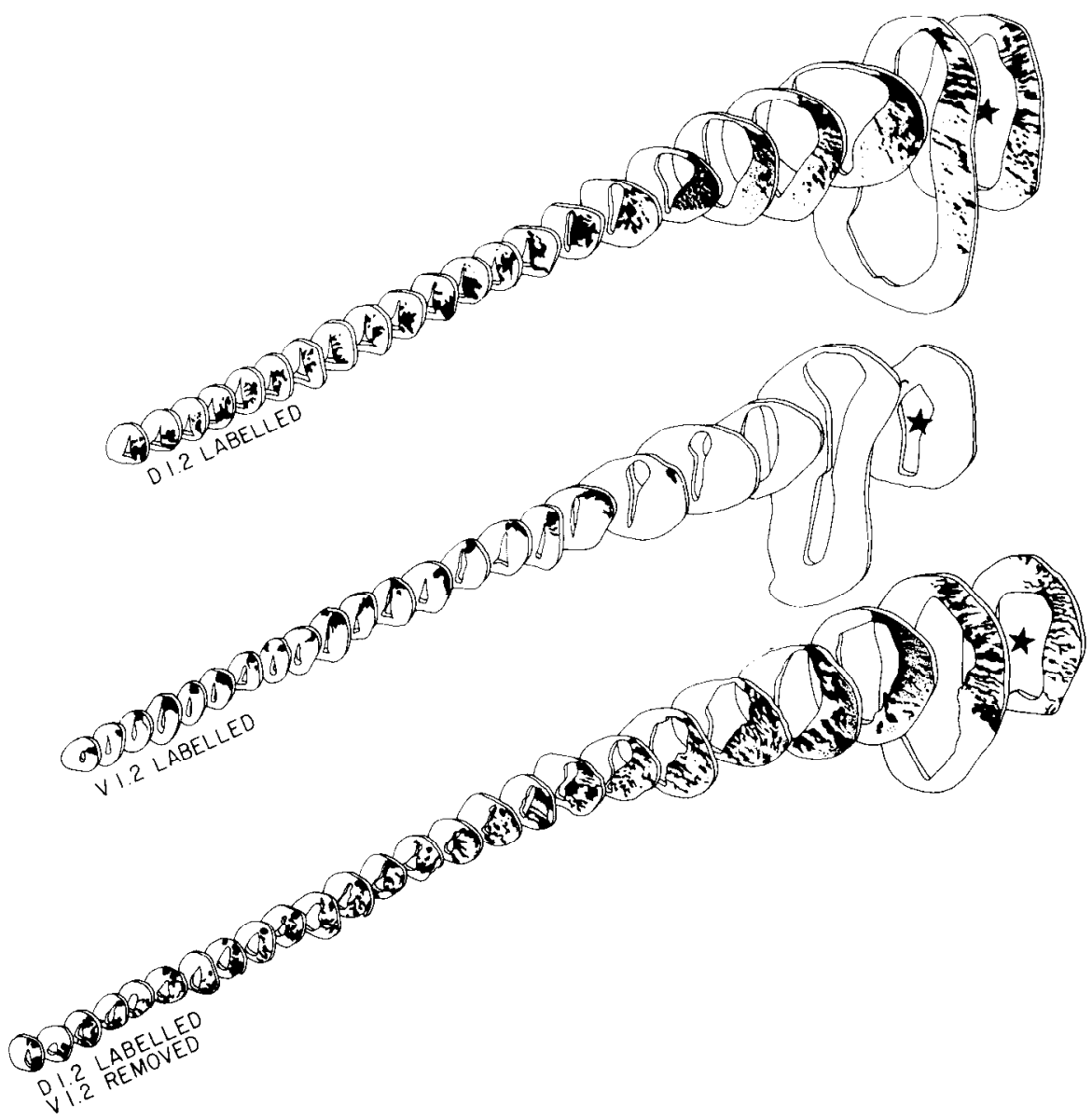

Figure 1. Serial section reconstructions (every fifth section) of the central nervous system in three Xenopus larvae that originated from type $\mathrm{X}$ embryos. Top, At stage 34 after injection of HRP into blastomere D1.2; middle, at stage 32 after injection of HRP into blastomere V1.2; bottom, at stage 33 after injection of HRP into blastomere D1.2 followed by removal of V1.2. The star is at the rostral end of the brain. Labeled cells are shown in black; unlabeled regions are shown in white. The diameter of the caudal section of the spinal cord is approximately $70 \mu \mathrm{m}$. 
D1.2 can substitute for V1.2 although they belong to different sets of progenitors which give rise to different types of neurons. Whether V1.2 is equally able to substitute for D1.2 is a more complex question that requires further investigation but that merits some discussion here. There is a considerable size disparity between the clonal domains of D1.2 and V1.2 in type X embryos (Fig. 1). Their descendants occupy adjoining regions in the spinal cord, but there is a disparity more rostrally because the V1.2 domain does not extend into the brain rostral to the rhombencephalon. Such a disparity is not found in the type $\mathrm{Y}$ cleavage pattern, where the V1.2 clonal domain extends into the dorsal mesencephalon and telencephalon and adjoins the D1.2 clonal domain along its entire length. This difference between the extent of the clonal domains in type $\mathrm{X}$ and type $\mathrm{Y}$ embryos allows an experimental test to be made of the hypothesis that substitution of one blastomere by another can occur only in the adjoining parts of their clonal domains. Thus, D1.2 can substitute completely for V1.2 in both type X and type Y embryos, but, if V1.2 is able to substitute for only the adjoining part of the D1.2 clonal domain, complete substitution would occur in type $\mathrm{Y}$ but not in type $\mathrm{X}$ embryos.

This paper is the first in a series designed to analyze regulation of presumptive neural cells in the early amphibian embryo. By focusing on the regulation of RohonBeard neurons and the Mauthner neuron, cells that originate in the gastrula and are easily identified and counted at early larval stages, I hope to approach studies in the amphibian embryo with the rigor which has been used to define and analyze the problem of regulation in invertebrate embryos.

\section{References}

Abercrombie, M. (1946) Estimation of nuclear population from microtome sections. Anat. Rec. 94: 239-247.
Hirose, G., and M. Jacobson (1979) Clonal organization of the central nervous system of the frog. I. Clones stemming from individual blastomeres of the 16-cell and earlier stages. Dev. Biol. 71: 191-202.

Holtfreter, J., and V. Hamburger (1955) Amphibians. In Analysis of Development, B. H. Willier, P. A. Weiss, and V. Hamburger, eds., pp. 230-296, Saunders, Philadelphia.

Hörstadius, S. (1950) The Neural Crest, Oxford University Press, London.

Hughes, A. F. (1957) The development of the primary sensory system in Xenopus laevis (Daudin). J. Anat. 91: 323-338.

Jacobson, M. (1981) Rohon-Beard neuron origin from blastomeres of the 16-cell frog embryo. J. Neurosci. 1: 918-922.

Jacobson, M., and G. Hirose (1981) Clonal organization of the central nervous system of the frog. II. Clones stemming from individual blastomeres of the 32 - and 64 -cell stages. J. Neurosci. 1: 271-284.

Lamborghini, J. L. (1980) Rohon-Beard cells and other large neurons in Xenopus embryos originate during gastrulation. J. Comp. Neurol. 189: 323-333.

Nakamura, O., and K. Kishiyama (1971) Prospective fates of blastomeres at the 32 cell stage of Xenopus laevis embryos. Proc. Jpn. Acad. 47: 407-412.

Nakamura, O., and H. Takasaki (1971) Analysis of causal factors giving rise to the organizer. I. Removal of polar blastomeres from 32 cell embryos of Xenopus laevis. Proc. Jpn. Acad. 47: 499-504.

Nakamura, O., H. Takasaki, and A. Nagata (1978) Further studies of the prospective fates of blastomeres at the 32-cell stage of Xenopus laevis embryos. Med. Biol. 56: 355-360.

Nieuwkoop, P. D., and J. Faber (1967) Normal Table of Xenopus laevis Daudin, Ed. 2, North-Holland, Amsterdam.

Spemann, H. (1938) Embryonic Development and Induction, Yale University Press, New Haven.

Sulston, J. E., and J. G. White (1980) Regulation and cell autonomy during postembryonic development of Caenorhabditis elegans. Dev. Biol. 78: 577-597.

Vogt, W. (1929) Gestaltungsanalyse am Amphibienkeim mit ortlicher Vitalfärbung. II Teil: Gastrulation und Mesodermbildung bei Urodelen und Anuren. Wilhelm Roux Arch. Entw. Mech. Org. 122: 667-669. 